\title{
Fuzzy Logic Based Control of Voltage and Reactive Power in Subtransmission System
}

\author{
Ana Paula M. Braga ${ }^{1}$, Ricardo Carnevalli ${ }^{2}$, Petr Ekel ${ }^{1}$, Marcelo Gontijo ${ }^{1}$, \\ Marcio Junges ${ }^{1}$, Bernadete Maria de Mendonça Neta ${ }^{2}$, \\ and Reinaldo M. Palhares ${ }^{3}$ \\ 1 Graduate Program in Electrical Engineering, \\ Pontifical Catholic University of Minas Gerais, \\ Ave. Dom José Gaspar, 500, 30535-610 - Belo Horizonte - MG, Brazil \\ anapaulamb@terra.com.br, ekel@pucminas.br, montalva@ig.com.br, \\ marcio.junges@jtech.eng.br \\ 2 Operation Coordination and Engineering Department, \\ Minas Gerais State Energy Company, \\ Ave. Barbacena, 1200, 30190-131 - Belo Horizonte - MG, Brazil \\ \{rcarne, bmmn\}@cemig.com.br \\ 3 Department of Electronics Engineering, \\ Federal University of Minas Gerais, \\ Ave. Antônio Carlos, 6627, 31270-010 - Belo Horizonte - MG, Brazil \\ palhares@cpdee.ufmg.br
}

\begin{abstract}
This paper presents results of research related to the second stage of the project dedicated to developing and employing the xOMNI system (software of the SCADA type). This stage is associated with the elaboration of the Energy Management System (EMS) for the subtransmission system of the Minas Gerais State Energy Company. One of the most important EMS functions is voltage and reactive power control. Its implementation is based on the use of fuzzy logic technology. Taking this into account, the paper covers the consideration of questions of building diverse types of sensitivity indices and forming production rules providing comprehensive and flexible solutions in conditions of regulated and deregulated environments. Some aspects of rational carrying out fuzzy logic procedures are considered as well.
\end{abstract}

\section{Introduction}

The Minas Gerais State Energy Company (CEMIG) attends more than 320,000 $\mathrm{km}$ of subtransmission and distribution networks and 340 subtransmission substations. These substations are controlled by distribution operation centers (DOCs). The xOMNI system (software of the SCADA type) [1] has been developed to realize this goal. The corresponding project has been divided into two stages. The first stage is associated with the SCADA phase and the second stage is related to the Energy Management System (EMS) phase.

The EMS functions that have already been developed are discussed in [1]. The main goal of this paper is to consider the function of voltage and reactive 
power control. Since its realization is based on integrating traditional numerical methods with fuzzy logic technology [2], the consideration includes questions of constructing diverse types of sensitivities (which serve for multi-attribute evaluating control actions efficiency), forming rules (that provide comprehensive and flexible solutions) as well as rational implementing fuzzy logic procedures.

\section{Fuzzy Logic Based Voltage and Reactive Power Control}

Critical investigations on fuzzy logic based voltage and reactive power control have been reported in [3]. They demonstrate that fuzzy logic is one of the most successful of today's technologies for elaborating sophisticated control systems. With its aid, complex requirements are implemented in systems with reasoning capabilities bound by a minimum of production rules that is reached on the basis of the interpolative nature of fuzzy set theory [2]. The approach [3] opened up an important direction in solving the problem of voltage and reactive power control. However, its application is limited by the following considerations:

- the approach is directed to solving the problem within the framework of simplified statement without considering an economical objective (minimization of losses) and restrictions on power-handling capability of system elements;

- the rules included in knowledge base are general, and questions of using rules, which reflect operational philosophy of a concrete system, are not considered;

- an important component of fuzzy modeling is the quality of models and their tuning, making possible improvement in control efficiency, that has not found consideration;

- availability of discrete operating values of regulating and compensating devices does not allow one to utilize the approach without modifications.

Overcoming of these aspects can permit one to use fuzzy logic for solving the problem under comprehensive statement.

\section{Construction of Sensitivity Indices}

The evaluation of influence of the control action of regulating or compensating device $j$ on the voltage change at bus $i$ is associated with the voltage sensitivity $S_{i j}^{V}$. In the system with $I$ controlled buses and $J$ buses with regulating and compensating devices, it is necessary to have a matrix $\left[S_{i j}^{V}\right], i=1, \ldots, I, j=1, \ldots, J$. In [3], its building is based on the use of system Jacobian matrices. However, deficient linearization accuracy in utilizing this approach allows one to apply it when perturbations are small. At the same time, our experience shows that experimental design [4] provides a means for rational building sensitivity models. Its use also permits one to eliminate from consideration actions at buses $j$, which have no influence on the voltage level at buses $i$, evaluate the adequacy of models, and, if necessary, change intervals of parameter varying to obtain adequate models. Finally, because the comprehensive solution needs sensitivities 
$\left[S_{k j}^{S}\right], k=1, \ldots, K, j=1, \ldots, J$ (reflecting the power flow change on line $k$ ) and $\left[S_{k j}^{S}\right]$, $k=1, \ldots, K, j=1, \ldots, J$ (reflecting the reactive power flow change on line $k$ allowing the estimation of loss increments [5]), their building is possible on the basis of the same computing experiments, which are necessary to obtain voltage sensitivities.

The full experiment is based on carrying out experiments with varying factors on two levels. It demands the performance of $2^{J}$ experiments to construct a model

$$
y=b_{0}+\sum_{j=1}^{J} b_{j} x_{j}+\sum_{\substack{j=1 \\ j<q}}^{J} b_{j q} x_{j}+\sum_{\substack{j=1 \\ j<q<r}}^{J} b_{j q r} x_{j} x_{q} x_{r}+\ldots .
$$

It is assumed that factors can take the minimum $x_{j}^{\prime}$ and maximum $x_{j}^{\prime \prime}$ values $\left(x_{j}^{\prime} \leq x_{j} \leq x_{j}^{\prime \prime}, j=1, \ldots, J\right)$ and are presented in the normalized form [4]. Thus, $x_{j}^{\prime}$ and $x_{j}^{\prime \prime}$ correspond to -1 and +1 , respectively.

A matrix for the full experiment with two factors is shown in Table 1.

Table 1. Matrix for the $2^{2}$ design

\begin{tabular}{cccccc}
\hline \multicolumn{3}{c}{ Factors } & \multicolumn{3}{c}{ Factor Product } \\
$n$ & $x_{0}$ & $x_{1}$ & $x_{2}$ & $x_{1} x_{2}$ & $y$ \\
\hline 1 & +1 & -1 & -1 & +1 & $y_{1}$ \\
2 & +1 & +1 & -1 & -1 & $y_{2}$ \\
3 & +1 & -1 & +1 & -1 & $y_{3}$ \\
4 & +1 & +1 & +1 & +1 & $y_{4}$ \\
\hline
\end{tabular}

Considering that $2^{J}>J+1$, data obtained in the full experiment have information excessiveness. It permits one to build linear models on the basis of fractional experiments. Their matrices are parts of the full experiment matrices and are obtained as a result of reducing a number of experiments in two, four, etc. times by replacing effects of little significance (for example, $\tilde{x}_{1} \tilde{x}_{2}$ in Table 1 ) by new parameters. The number of replacements $g$ defines the $2^{J-g}$ design. For example, to estimate coefficients of a model $y=b_{0}+b_{1} x_{1}+b_{2} x_{2}+b_{3} x_{3}$ we have to perform eight experiments, although it is enough to perform four experiments in accordance with the $2^{3-1}$ design coinciding with the $2^{2}$ design if $\tilde{x}_{1} \tilde{x}_{2}=\tilde{x}_{3}$.

A traditional technique for evaluating experiment results includes several stages [4]. These stages are common if we can perform parallel experiments in corresponding factorial space points. If we speak about computing experiments with a model, this circumstance has a significant impact. One way around this problem is discussed in [5].

The described approach was tested on the CEMIG subtransmission system. Simulation results show its high computing performance: it was necessary less than 6 min. of computer (Pentium IV $1.8 \mathrm{GHz}$ with RAM of $512 \mathrm{MB}$ ) time (with executing other tasks) to calculate all types of sensitivities for the CEMIG subtransmission system (72 buses with regulating and compensating devices) on the basis of the $2^{72-65}$ design. This permits one to use the approach without system decomposition. 


\section{Rules and Linguistic Variables}

Comprehensive statement of the problem of voltage and reactive power control is to cover the following control hierarchy levels: (A) all restrictions are observed, and it is necessary to minimize losses, (B) restrictions on bus voltage levels and/or on power-handling capability of system elements are not observed, but the violations can be eliminated, and (C) restrictions on bus voltage levels and/or on power-handling capability of system elements are not observed, and the violations cannot be eliminated.

The approach [3] provides corrective control and is based on the use of rules of the following type:

(I) IF bus voltage violates the operational limit

AND a controller is available for effective voltage control adjusting its output

AND there is adequate margin of output adjustment to eliminate the restriction violation

THEN increase (decrease) the controller output.

Other works related to using knowledge based methodology also distinguish the phases of corrective control and loss minimization with eliminating the last objective from a knowledge based control loop. However, more comprehensive and flexible solutions may be obtained if knowledge base includes the following type of rules for the hierarchy level (B):

(II) IF bus voltage violates the operational limit

AND a controller is available for effective voltage control adjusting its output

AND there is adequate margin of output adjustment to eliminate the restriction violation

AND the controller is available for loss reduction (increase)

THEN increase (decrease) the controller output.

It should be pointed out that the hierarchy level $(\mathrm{A})$ is particular in relation to the level (B). The level (C) is also reduced to the level (B) if priorities are introduced, for example, for buses under infeasibility minimization conditions.

Generally, the use of the rules of the type (II) provides solutions different from the results obtained on the basis of the rules of the type (I). Our experience shows that using the rules of the type (II), it is possible to reduce losses even at the phase of corrective control.

Since the rules (I) and (II) reflect general strategies, each of them is presented by a set of rules defined by fuzzy values [2] of linguistic variables, which are control efficiency, adequate margin, and loss increment as the input variables and utilization intensity as the output variable.

The reactive power planning and operation are important functions of ancillary service markets. The philosophy of [6] justifies the expediency of their structuring within the framework of preparation markets and actuation markets. 
In operating actuation markets, a function of observing restrictions on reactive power levels is to be realized. The restrictions may be considered in a traditional way as the restrictions on voltage levels with using $\left[S_{k j}^{Q}\right], p=1, \ldots, P, j=1, \ldots, J$. However, there is another way associated with considering the restrictions on reactive power levels on the basis of minimizing

$$
D=\left(\sum_{p=1}^{P} Q_{p}^{0}\left|Q_{p}-Q_{p}^{0}\right|\right) / \sum_{p=1}^{P} Q_{p}^{0},
$$

which reflects the weighted average magnitude of deviations of reactive power (WAMDRP) from their desirable levels $Q_{p}^{0}, p=1, \ldots, P$.

Applying (2), it is possible to realize observing the desirable reactive power levels with using $\left[S_{j}^{D}\right], j=1, \ldots, J$ and rules of the following type:

(III) IF bus voltage violates the operational limit

AND a controller is available for effective voltage control adjusting its output

AND there is adequate margin of output adjustment to eliminate the restriction violation

AND the controller is available for reduction (rise) of WAMDRP

AND the controller is available for loss reduction (increase)

THEN increase (decrease) the controller output.

\section{Fuzzy Logic Procedures}

The collection of rules may be presented by the following fuzzy algorithm:

$$
\begin{aligned}
& \text { IF } x_{1}=a_{11} \ldots \text { AND } x_{k}=a_{1 k} \text { AND } \ldots \text { AND } x_{m}=a_{1 m} \text { THEN } y=b_{1} \text { ELSE } \\
& \ldots \\
& \text { IF } x_{1}=a_{p 1} \ldots \text { AND } x_{k}=a_{p k} \text { AND } \ldots \text { AND } x_{m}=a_{p m} \text { THEN } y=b_{p} \text { ELSE } \\
& \quad \ldots \\
& \text { IF } x t_{1}=a_{t 1} \ldots \text { AND } x_{k}=a_{t k} \text { AND } \ldots \text { AND } x_{m}=a_{t m} \text { THEN } y=b_{t}, \quad
\end{aligned}
$$

where $a_{p k}, p=1, \ldots, t, k=1, \ldots, m$ and $b_{p}, p=1, \ldots, t$ have estimates $A_{p k}$ and $B_{p}$ with the membership functions $\mu_{A_{p k}}\left(x_{p k}\right)$ and $\mu_{B_{p}}(y)$, respectively.

The collection (3) is equivalent to the $(m+1)$-dimensional implication relation matrix $R$ [2]. Its use permits one to carry out inference under arbitrary values of $x_{1}=a_{1}^{\prime}, x_{2}=a_{2}^{\prime}, \ldots, x_{m}=a_{m}^{\prime}$ on the basis of

$$
\mu_{B^{\prime}}(y)=\mu_{A_{1}}\left(x_{1}\right) \circ \mu_{A_{2}}\left(x_{2}\right) \circ \ldots \circ \mu_{A_{m}}\left(x_{m}\right) \circ R,
$$

where the symbol o defines the type of composition.

To avoid manipulations with multidimensional matrices in accordance with (4), it is appropriate to introduce [7] the possibility measure " $a_{p k}$ is $a_{k}^{\prime} "$ :

$$
\operatorname{Poss}\left(a_{p k} \mid a_{k}^{\prime}\right)=\vee_{x_{k}} \mu_{A_{p k}}\left(x_{k}\right) \wedge \mu_{A_{k}^{\prime}}\left(x_{k}\right) \text {. }
$$


Using (5), it is possible to define

$$
\operatorname{Poss}\left(a_{p 1}, \ldots, a_{p k}, \ldots, a_{p m} \mid a_{1}^{\prime}, \ldots, a_{k}^{\prime}, \ldots, a_{m}^{\prime}\right)=\wedge_{k} \operatorname{Poss}\left(a_{p k} \mid a_{k}^{\prime}\right),
$$

which reflects the possibility measure that the constituent concept described by $a_{p 1}, \ldots, a_{p k}, \ldots, a_{p m}$ is constituent concept characterized by $a_{1}^{\prime}, \ldots, a_{k}^{\prime}, \ldots, a_{m}^{\prime}$.

Considering (5) and (6), it is possible to build

$$
\mu_{B^{\prime}}(y)=\vee_{p}\left[\mu_{B_{p}}\left(y_{p}\right) \wedge \wedge_{k} \operatorname{Poss}\left(a_{p k} \mid a_{k}^{\prime}\right)\right],
$$

which is equivalent to (4).

The estimates of the computational complexity in carrying out fuzzy inference on the basis of (4) and (7) are given in [8]. They demonstrate the efficiency of realizing fuzzy inference on the basis of $(7)$. This as well as a convenience of implementing (7) explains its application.

\section{Conclusion}

Questions of using fuzzy logic technology for voltage and reactive power control have been considered. An approach based on experimental design has been applied to build diverse types of sensitivities to evaluate efficiency of control actions and construct rules included in knowledge base. Diverse types of rules applicable to regulated and deregulated environments have been presented. Some aspects of rational carrying out fuzzy logic procedures have been discussed as well.

\section{References}

1. Morra, J.L.T., Carnevalli, R.L.J., Mendonça Neta, B.M., Costa, S.T.M., Garcia, A.V., Ekel, P.I.: xOMNI Sistema SCADA/EMS, Revista CIER 41 (2002) 42-48.

2. Tsoukalas, L.H., Uhrig, R.E.: Fuzzy and Neural Approaches in Engineering. Wiley, New York (1997).

3. Yokoyama, R., Nimura, T., Nakanishi, Y.: A Coordinated Control of Voltage and Reactive Power by Heuristic Modeling and Approximate Reasoning. IEEE Trans. Power Systems 8 (1993) 636-645.

4. Box, G.E.P., Hunter, W.G., Hunter, J.S.: Statistics for Experiments. An Introduction to Design, Data Analysis and Model Building. Wiley, New York (1978).

5. Ekel, P.Ya., Junges, M.F.D., Morra, J.L.T., Paletta, F.P.G.: Fuzzy Logic Based Approach to Voltage and Reactive Power Control in Power Systems. Int. J. of Computer Research 11 (2002)159-170.

6. Kaye, R.J., Zammit, M.A.B., Hill, D.J.: Co-ordinated Spot and Ancillary Service Market to Optimise Power Systems Security. Proc. Int. Symp. on Bulk Power Systems Dynamics and Control IV: Restructuring. Santorini (1998) 539-544.

7. Sanchez, E.: Resolution of Eigen Fuzzy Sets Equations. Fuzzy Sets and Systems 1 (1978) 69-74.

8. Ekel, P., Popov, V.: Fuzzy Set Theory and Problems of the Design and Control of Power Systems and Subsystems, Proc. 4th IEEE Conf. on Control Applications (1995) 46-51. 